\title{
A Precision Signal Amplifier for Improved Imaging of Insulating Samples using Variable Pressure SEM
}

\author{
W.R. Knowles*, M. Kliment*, R. Schroemges* \\ *FEI Company, 5350 NE Dawson Creek Drive, Hillsboro, OR 97124
}

A high gain and bandwidth, low noise amplifier has been developed for improved imaging in environmental and variable pressure SEM. The calibrated, precision amplifier is also being used in quantitative studies of the physics of gaseous electron detectors.

Historically, most SEM imaging has been done using the scintillator/photomultiplier configuration employed in the Everhart-Thornley detector (E-T) [1]. As the photomultiplier gain is very high (up to $10^{6}$ ), only a very simple electronic amplifier is needed to convert its output to the level required by the signal acquisition system. Hence, the performance is dominated by the detector itself and the photomultiplier (i.e., not by an electronic amplifier). Most SEMs now have a capability for secondary electron imaging of insulating samples using gas amplification inside the specimen chamber. The physics of the gas amplification process has been described in the literature [2]. However, the maximum achievable gas gain is on the order of $10^{3}$, and a high gain electronic amplifier is needed to amplify the gas-pre-amplified imaging signal. Optimum detector configurations also require that this amplifier be connected to a high voltage anode. Consequently, an advanced amplifier design is required so as not to degrade the imaging performance (i.e., signalto-noise ratio, bandwidth and headroom). We have developed a low noise, precision amplifier that has very high sensitivity and wide bandwidth.

\section{Amplifier Characteristics}

The sensitivity of 1nA allows imaging at the lowest beam currents used in a tungsten variable pressure SEM (since the electron signal is pre-amplified in the gas cascade). The amplifier noise is negligible compared to the shot noise of the secondary electron signal even at incident beam currents as low as $1 \mathrm{pA}$.

The amplifier collects the signal from the anode floating at up to $+800 \mathrm{~V}$. Any electrical leakage to ground would generate a spurious signal offset and extra noise. By careful design and construction, the leakage is negligible compared to the $1 \mathrm{nA}$ minimum signal input. Leakage resistances of more than $10^{13}$ ohms are routinely achieved.

The amplifier and signal connections have special measures to avoid the pickup of interference from the environment or other electronics in the microscope, even at the extremely high gain of this amplifier.

The amplifier bandwidth is $3.5 \mathrm{MHz}$ which allows imaging at the fastest scan rates for easy navigation around the sample.

The gain is adjustable over a wide range, covering all normal imaging conditions. This allows the anode voltage to be optimized for charge control. The high precision of the gain control allows very effective implementation of automatic brightness and contrast. 
A novel architecture allows the user to expand the contrast of the image in the preamplifier to generate high contrast images, even with the low inherent contrast that is typical of uncoated insulating samples. Contrast expansion can be as large as 50x, depending on operating conditions.

In addition to the improvements for the user, this amplifier is a very effective tool for scientific study of the gaseous detection process. A novel gaseous detector has been developed for imaging insulating samples at very high magnification using an immersion objective lens [3]. The new amplifier was an essential tool in the characterization and optimization of this "Helix" detector.

We are able to accurately measure the gas amplified signal over a very wide range of currents ( $1 \mathrm{nA}$ to $250 \mathrm{nA}$ ) with an absolute accuracy of better than $5 \%$ over the gain range. Figure 1 shows the gain of the Helix detector under typical operating conditions, characterized using the amplifier.

The response speed of the signal from the gas cascade is limited by the ion drift time within the detector volume [4]. The response is slower than the traditional E-T detector. Some literatures suggest that the speed is in the range of hundreds of microseconds. We suspect that these values are a result of amplifier limitations or poor detector geometry, rather than the gas physics. The fast speed of the new amplifier (100ns risetime) allowed accurate measurements of the Helix detector response. Typical values are around one microsecond depending on the exact detector geometry. To illustrate this, the smear-free Helix detector image shown in Fig 2 was acquired using a pixel dwell time of $800 \mathrm{~ns}$.

\section{References}

[1] T.E. Everhart and R.F.M. Thornley, Journal of Scientific Instruments, Vol. 37, 246-248,(1960)

[2] B. L. Thiel, et al,. J. Microsc.-Oxf. 187: 143-157 (1997).

[3] B. L. Thiel, et al., J. Appl. Phys., submitted. (2005).

[4] Mohan, A., N. Khanna, et al., Scanning 20: 436-441. (1998).

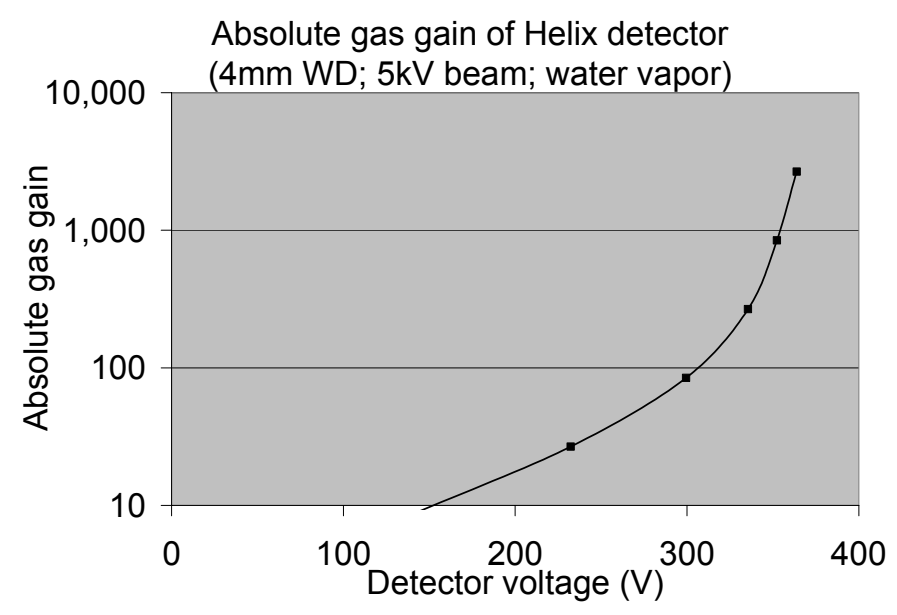

Figure 1. Gas gain of the Helix detector under typical operating conditions

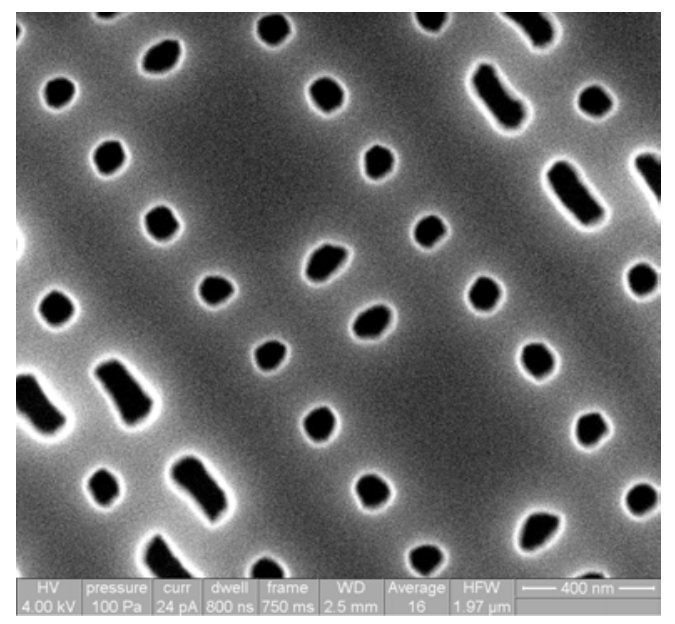

Figure 2. Image of a $\mathrm{SiN} / \mathrm{Si}$ multilayer obtained using a pixel dwell time of $800 \mathrm{~ns}$ (16 frame average). 